\title{
Phyllotaxis bionics for vortex nanosieves
}

Min Gu (id ${ }^{1,2 \otimes}$, Yilin Hua ${ }^{1,2}$ and Xinyuan Fang $\mathbb{B}^{1,2}$

\section{eLight, 1(5), 2021}

https://doi.org/10.1186/s43593-021-00005-9

Simultaneous generation of multiple optical vortex (OV) lies at the heart of the application for orbital angular momentum (OAM) multiplexing both in classical and quantum domains. Previous structure with segmented or interleaved functional sub-areas in a single nano-device has been developed at the cost of its compactness and channel capacity. Back-to-nature design inspired by the spiral phyllotaxis pattern in this work offers a fresh way to fabricate a truly space-degenerated nanoscale multipleOVs-generator, namely vortex nanosieves. Both freespace and plasmonic near-field OAM multiplexed OVs are produced with two different forms of the phyllotaxisinspired patterns, which are proved by rigorous mathe- matical language and completely consistent experimental results. Except for the structural degeneracy of the device, the OAM modes extracted from the vortex nanosieves are controllable according to the internally embedded multiple spirals, which is particularly suitable for the highorder OAM modes generation. Furthermore, the OAM multimode output displays a concentric-ring type naturally with negligible misalignment. These superiorities make the phyllotaxis-inspired vortex-nanosieves device a programmable multi-OAM-modes generator and multiplexer, which will facilitate research in both on-chip photonic devices and OAM information optics.

Received: 31 August 2021 Accepted: 9 September 2021

Published online: 22 September 2021 